\title{
Systematic literature review investigating whether methotrexate causes chronic pulmonary fibrosis
}

\author{
Authors: Ed Quah, Constanta Amoasii, Tareg Mudawi and Julie Dawson
}

\section{Introduction}

Methotrexate is a treatment widely used in many medical conditions. It is highly effective especially in conditions such as rheumatoid arthritis (RA) and psoriasis. Methotrexate is associated with a potentially fatal acute methotrexate pneumonitis. In regards to chronic lung complications, three case reports with a total of four patients have suggested a link between methotrexate and chronic lung fibrosis. ${ }^{1-3}$ Subsequent longitudinal studies have refuted this claim. ${ }^{4,5}$ The aim in this study is to undertake a systematic literature review to evaluate the published evidence on whether methotrexate causes chronic interstitial pulmonary fibrosis in humans.

\section{Methods \\ The preferred reporting items for systematic reviews and meta- analyses (PRISMA) checklist for systematic reviews was followed. The review was registered with Prospero. Electronic searches were performed using scientific literature databases for articles relating to methotrexate use and chronic pulmonary fibrosis. Literature searches were performed by two investigators in two stages. Non- English, non-human and all other methotrexate complications apart from interstitial lung fibrosis were excluded from this study. Data collected were subsequently separated into two groups (one supporting and one rejecting the claim of methotrexate-induced pulmonary fibrosis). Articles in each group were analysed based on their level of evidence using Oxford (UK) and Downs and Black quality scoring. Risk of bias was assessed with the risk of bias in non-randomized studies of interventions (ROBINS-I) assessment tool.}

\section{Results and discussion}

A total of 22 articles met the inclusion criteria, 11 articles were found to support the claim that methotrexate causes pulmonary fibrosis and another 11 found otherwise. It was found that $63 \%$ the articles from the supporting groups were published before the year 2000. Articles in the supporting papers are of a low Downs and Black score (<6/27) and are at a serious-to-critical level of bias. They contain three case reports/series and seven review articles.

Authors: St Helens and Knowsley Trust Hospitals, UK
With regards the articles in the rejecting group, they are higher scoring on the Downs and Black score and have only low-tomoderate risk of bias. They contain cohort studies, one review article and two meta-analyses of randomised controlled trials of methotrexate. The study populations in the meta-analyses contained 1,630 and 8,584 patients. The most recent cohort study where 52/78 RA-interstitial lung disease (ILD) patients were treated with methotrexate: methotrexate treatment was actually associated with a statistically significant improved survival.

\section{Conclusion}

Our systematic literature review found high-quality unbiased evidence that methotrexate does not cause chronic pulmonary fibrosis in humans. In patients with RA, chronic pulmonary fibrosis is due to the underlying disease and not methotrexate. It is important to now address this across all disciplines and reject the hypothesis that methotrexate causes chronic pulmonary fibrosis as it is resulting in patients having their methotrexate therapy withdrawn without any quality evidence base to support that clinical decision. It is possible that methotrexate treatment may actually improve outcomes in RA-ILD patients which is an area for further research. ${ }^{6}$

\section{Conflict of interest statement}

None declared.

\section{References}

1 Bedrossian CW, Miller WC, Luna MA. Methotrexate-induced diffuse interstitial pulmonary fibrosis. South Med J 1979;72:313-8.

2 Kaplan RL, Waite DH. Progressive interstitial lung disease from prolonged methotrexate therapy. Arch Dermatol 1978;114:1800-2.

3 Phillips TJ, Jones DH, Baker H. Pulmonary complications following methotrexate therapy. J Am Acad Dermatol 1987;16:373-5.

4 Dawson JK, Graham DR, Desmond J, Fewins HE, Lynch MP. Investigation of the chronic pulmonary effects of low-dose oral methotrexate in patients with rheumatoid arthritis: a prospective study incorporating HRCT scanning and pulmonary function tests. Rheumatology (Oxford) 2002;41:262-7.

5 Provenzano G. Chronic pulmonary toxicity of methotrexate and rheumatoid arthritis. Rheumatology (Oxford) 2003:42:802-3.

6 Rojas-Serrano ], Herrera-Bringas D, Pérez-Román DI et al. Rheumatoid arthritis-related interstitial lung disease (RA-ILD): methotrexate and the severity of lung disease are associated to prognosis. Clin Rheumatol 2017;36:1493-500. 